\title{
Escuela y diversidad lingüística: estudio de las actitudes lingüísticas de los futuros docentes valencianos
}

\section{School and linguistic diversity: study of the language attitudes of future Valencian teachers}

\author{
M-Begoña Gómez-Devís \\ Universitat de Valencia* \\ mabegode@uv.es \\ José Buzón \\ Universitat de Valencia \\ buzon_jos@gva.es \\ Miquel Alandete-Ballester \\ Valencian International University \\ miquel.alandete@campusviu.es
}

\begin{abstract}
The article analyses the linguistic attitudes of future Valencian teachers in a bilingual speaking community characterised by mainstream contact between the Catalan and Spanish languages. This research focuses on the language attitudes of a group of speakers who share a specific set of traits: age, education, profession and socioeconomic status. Special attention is given to attitudes towards four linguistic varieties used by Valencian speakers in their regular interactions: standard Spanish (CS), colloquial Spanish - nonstandard - (CNS), standard Valencian (VS) and vernacular Valencian or apitxat (VNS).
\end{abstract}

Keywords: language contact, attitude, teacher training, ideology, Valencia

${ }^{*}$ Referencia del grupo de investigación Conocimiento didáctico del contenido escolar: fundamentos del análisis y la acción didáctica del profesorado (CDC) de la Universitat de València. 


\section{INTRODUCCIÓN}

El paisaje lingüístico de las sociedades modernas ya no se distingue como multilingüe, sino además plurilingüe; es decir, implica la comprensión del idioma del otro $\mathrm{y}$, por ello e inevitablemente, conlleva una determinada actitud ante el mismo. La ordenación de esta convivencia lingüística, más o menos conflictiva, es tarea y responsabilidad de distintas instituciones que ejercen su influencia en múltiples esferas; y entre ellas se considera de especial atención la educativa.

La peculiar situación del contacto entre español y catalán - en su variedad valenciano - no es reciente ni desconocida. De hecho, estudiar la comunidad bilingüe de Valencia resulta sumamente atractivo, pues se trata de una comunidad de habla en la que el bilingüismo social se caracteriza por su intensidad (uso interactivo frecuente en las dos lenguas), extensión (número elevado de hablantes bilingües) y estabilidad (coexistencia durante siglos).

La contribución que aquí se presenta se centra en el estudio de un colectivo específico, maestros y maestras en formación (Facultat de Magisteri, Universitat de València) que comparten rasgos como la edad, profesión, estudios realizados, posición social, etc. Esto nos permitirá estudiar el modo en que se configuran actitudes lingüísticas diversas en un medio básicamente homogéneo, lo cual constituye un importante complemento de los resultados obtenidos en investigaciones con una muestra general de la comunidad lingüística estudiada.

En este sentido, el estudio sociolingüístico del valenciano en la comunidad universitaria cuenta ya con algunos trabajos centrados en los comportamientos lingüísticos de sus estudiantes, profesorado y personal de servicios (Aparici \& Castelló 2011; Casesnoves \& Mas 2016, 2017) que corroboran en buena parte de los indicadores analizados, especialmente aquellos que miden las competencias lingüísticas y evalúan el nivel de uso, unos resultados por encima de la media de la población en general. No obstante, para la investigación en ciencias sociales (y particularmente en educación) resulta prioritario conocer las opiniones y comportamientos de aquellos que en breve ejercerán la profesión docente inmersos en un contexto educativo bilingüe o, en el mejor de los casos, plurilingüe.

Así, el principal reto de esta investigación es descubrir las actitudes lingüísticas de los futuros profesionales de la docencia (último curso de la titulación de Grado en Maestro en Educación Primaria) hacia las cuatro variedades presentes en la interacción lingüística de la comunidad de habla bilingüe de Valencia: castellano estándar (CS), castellano popular - no estándar - (CNS), valenciano estándar (VS) y valenciano central apitxat - vernáculo - (VNS). Gracias a una serie de análisis cuantitati-vos es posible desgranar dicho reto en tres objetivos específicos:

a) primero: conocer las evaluaciones obtenidas en las dimensiones cognoscitiva (competencia personal, estatus, prestigio profesional, interacción comunica- 
tiva, tendencia ideológica) y afectiva (atractivo personal y social, endogrupo, solidaridad) con objeto de conocer el papel que desempeña cada modalidad lingüística;

b) segundo: establecer cuál es la variedad lingüística identificada como más apropiada para distintos ámbitos de uso, dato que permitirá establecer si existe un bilingüismo diglósico (cierta distribución funcional de las dos lenguas en contacto);

c) tercero: comprobar la influencia de los factores sociales (sexo, lengua materna, lengua habitual y residencia) en la percepción y valoración de las distintas variedades dialectales.

En cuanto a las hipótesis de trabajo cabe señalar que se han formulado tomando como referencia los resultados de trabajos recientes sobre nuestro objeto de estudio (AVL 2005; Agulló 2011; Aparici \& Castelló 2011; Casesnoves 2012; Gómez Molina 2013; Casesnoves \& Mas 2016, 2017; Buzón et al. 2017; Mas 2018).

La primera hipótesis considera los valores del estatus y la solidaridad como factores que determinan ciertas correlaciones. Las variedades estándar aparecerán vinculadas al estatus, medios de comunicación y poder; y las no estándar, a los valores intragrupales de solidaridad y lealtad lingüística.

Para la segunda hipótesis nos centramos en los índices de percepción positiva del valenciano frente al castellano en determinados aspectos instrumentales, prestigio lingüístico y valor integrativo. Al tratarse de un colectivo cuyo perfil formativo y profesional está claramente posicionado, los resultados obtenidos corroborarán que estos jueces valoran esta modalidad más positivamente que la población en general.

La tercera hipótesis considera el proceso de normalización lingüística del valenciano en el ámbito educativo ${ }^{1}$, iniciado en 1983 (Ley de Uso y Enseñanza del Valenciano) y reformulado en distintas regulaciones legislativas hasta la actual Ley de Plurilingüismo de la Generalitat Valenciana (2018). Recientes investigaciones indican que dicho proceso no solo no avanza, sino que la sustitución lingüística del valenciano por el castellano se va consolidando progresivamente, consecuencia tanto de la política lingüística aplicada en los últimos veinte años como de la escasa implicación subjetiva y presión social en el uso del valenciano.

Por último, los factores sociales con mayor peso probabilístico en las actitudes lingüísticas manifestadas por los estudiantes de magisterio serán, en orden decreciente: lengua habitual, sexo, lengua materna y residencia.

\footnotetext{
${ }^{1}$ Tal y como recoge el Estatuto de Autonomía de la Comunidad Valenciana (Ley Orgánica 1982), Título I, artículo 6: (1) La lengua propia de la Comunidad Valenciana es el valenciano; (3) La Generalitat garantizará el uso normal y oficial de las dos lenguas y adoptará las medidas necesarias para asegurar su conocimiento; (5) Se otorgará especial protección y respeto a la recuperación del valenciano; (6) La ley establecerá los criterios de aplicación de la lengua propia en la Administración y la Enseñanza.
} 


\section{ACTITUDES LINGÜÍSTICAS Y PROFESIÓN DOCENTE}

La comunidad científica actual acepta que investigar las actitudes lingüísticas contribuye a un mejor conocimiento de la variación intralingüística, permite jerarquizar las variedades diatópicas de una lengua, propicia la evaluación de variedades lingüísticas en una comunidad de habla bilingüe o multilingüe y resulta imprescindible para una buena planificación lingüística. No obstante, si bien las consideraciones sobre su utilidad son unánimes, existe cierta controversia sobre los límites epistemológicos que comporta su estudio, así como su naturaleza y estructura.

Sin ánimo de menoscabar el marco teórico de la disciplina sociolingüística, los autores de este trabajo suscribimos la propuesta de López Morales (1989: 235) y entendemos la actitud como la acción o reacción misma - conducta - la aceptación o el rechazo de un hecho lingüístico - y, por tanto, el uso o desuso de una variante, de una variedad o de una lengua - que se produce por las creencias, favorables o adversas, hacia el hecho en cuestión; tales creencias provienen de los conocimientos lingüísticos y sociolingüísticos del hablante, es decir, de su conciencia sociolingüística - dimensión cognoscitiva - o de percepciones y consideraciones relacionadas con la subjetividad y la emoción - dimensión afectiva - propias y de su comunidad de habla.

Desde esta perspectiva y citando al mismo autor, el engranaje sociolingüístico puede explicarse de la manera que sigue: el conocimiento del funcionamiento del uso lingüístico, así como las percepciones afectivas hacia él por parte de los miembros de una comunidad, produce ciertas creencias sobre ese uso que, si son positivas, le confieren estatus y pueden provocar actitudes positivas en el individuo quien, por tanto, lo acepta y lo emplea; pero si son negativas lo estigmatizan, dando como resultado la aparición de actitudes negativas que impiden que los miembros de la comunidad lo hagan suyo.

Esta concepción nos permite el estudio empírico de las actitudes sociolingüísticas de los hablantes a partir de sus percepciones y creencias hacia dimensiones que tienen que ver, por un lado, con su conocimiento - componente cognoscitivo: conciencia sociolingüística basada en prestigios abiertos, sociales y lingüísticos $-\mathrm{y}$, por otro, con su afectividad - componente afectivo: sentimientos provenientes de prestigios psicosociales abiertos o encubiertos, que se relacionan con gustos, identidad, solidaridad, lealtad, etc.

El procedimiento metodológico implementado se basa en valoraciones directas que nos informan de la predisposición del hablante a utilizar o no una determinada modalidad de lengua o una lengua en situación de contacto, o de su predisposición a enseñarla o aprenderla. Como indican Buzón et al. (2017) la percepción de un estímulo (fragmento discursivo en nuestro caso) "pone en marcha una reacción evaluativa (favorable/desfavorable) en un contexto de consideraciones y conocimientos compartidos (social), resultado de los procesos cognoscitivo y afectivo 
(individual)" (p. 65). Pero al ajustar el punto de mira en la profesión docente, obtener información sobre la percepción de las modalidades lingüísticas disponibles cobra especial sentido. Rescatamos del pasado las palabras de Rojo (1979) :

En el caso de los maestros no es posible olvidar que estamos ante quienes desempeñan uno de los papeles protagonistas del proceso educativo (...). De un lado, la actitud del maestro hacia las dos o más lenguas implicadas (y hacia quienes las hablan) puede determinar su postura con respecto a cuestiones académicas relevantes $y$, en consecuencia, las expectativas de los futuros alumnos y de sus familiares. (...) De otra parte, todo intento de planificación lingüística pasa irremediablemente (al menos en Galicia) por la escuela, lo cual hace absolutamente imposible trazar una línea de actuación, cualquiera que sea, sin conocer el modo de pensar de quienes han de ponerla en práctica (p. 131).

\section{PLANTEAMIENTO METODOLÓGICO}

De acuerdo con los postulados sociolingüísticos, este estudio asume la necesidad de aplicar la metodología idónea a los fines de la investigación (cfr. Moreno Fernández 1990; López Morales 1994), así como las observaciones de Giles y Ryan (eds.) (1982: 208-223) sobre los jueces evaluadores y el análisis de las actitudes respecto de los procesos psicológicos vinculados con ellas ${ }^{2}$.

\subsection{La medición de creencias y actitudes: Recogida de materiales}

Para la obtención de los datos se ha preparado una prueba que, partiendo de pares falsos o máscaras (matched-guise technique) permite recabar datos sobre la valoración que los estudiantes de magisterio efectúan de las variedades (estándar y no estándar) de las lenguas cooficiales en la Comunitat Valenciana.

La recogida del material se realiza durante el curso académico 2015-2016 mediante entrevista directa en diferentes sesiones a un número reducido de participantes en cada sesión (pequeño grupo) hasta un total de 37 individuos $^{3}$. El procedimiento en todas las sesiones es el siguiente: audición de un texto leído (soporte digital) en una de las modalidades lingüísticas (CS, VS, CNS, VNS) y, seguidamente, realización de la encuesta. Una vez todos los estudiantes han anotado sus respuestas se pasa a la siguiente modalidad. Con objeto de evitar el sesgo que

\footnotetext{
${ }^{2} \mathrm{Se}$ ha analizado la actitud mediante técnicas indirectas (test de reacciones subjetivas ante estímulos discursivos leídos).

${ }^{3}$ Se trata, por tanto, de un muestreo no aleatorio y por conveniencia.
} 
pudiera producir el orden de presentación de las cuatro grabaciones se ha procedido a ordenarlas de modo aleatorio en cada uno de los pases. La duración de la encuesta completa es de una hora aproximadamente y se trabaja únicamente con audiciones de mujeres, a fin de controlar que el timbre masculino o femenino de la voz evaluada no interfiera en las valoraciones.

\subsection{La muestra}

Los individuos que han evaluado las cuatro modalidades lingüísticas las conocen bien, ambas lenguas son materia de estudio a lo largo de su etapa educativa (colegio, instituto y universidad) $\mathrm{y}$, aunque no todos ellos las usan con regularidad, representa el instrumento de interacción cotidiana en esta zona geográfica y pueden juzgarlas mostrando sus preferencias.

Tabla 1. Distribución de los hablantes por variables sociales (residencia, sexo, lengua habitual y lengua materna)

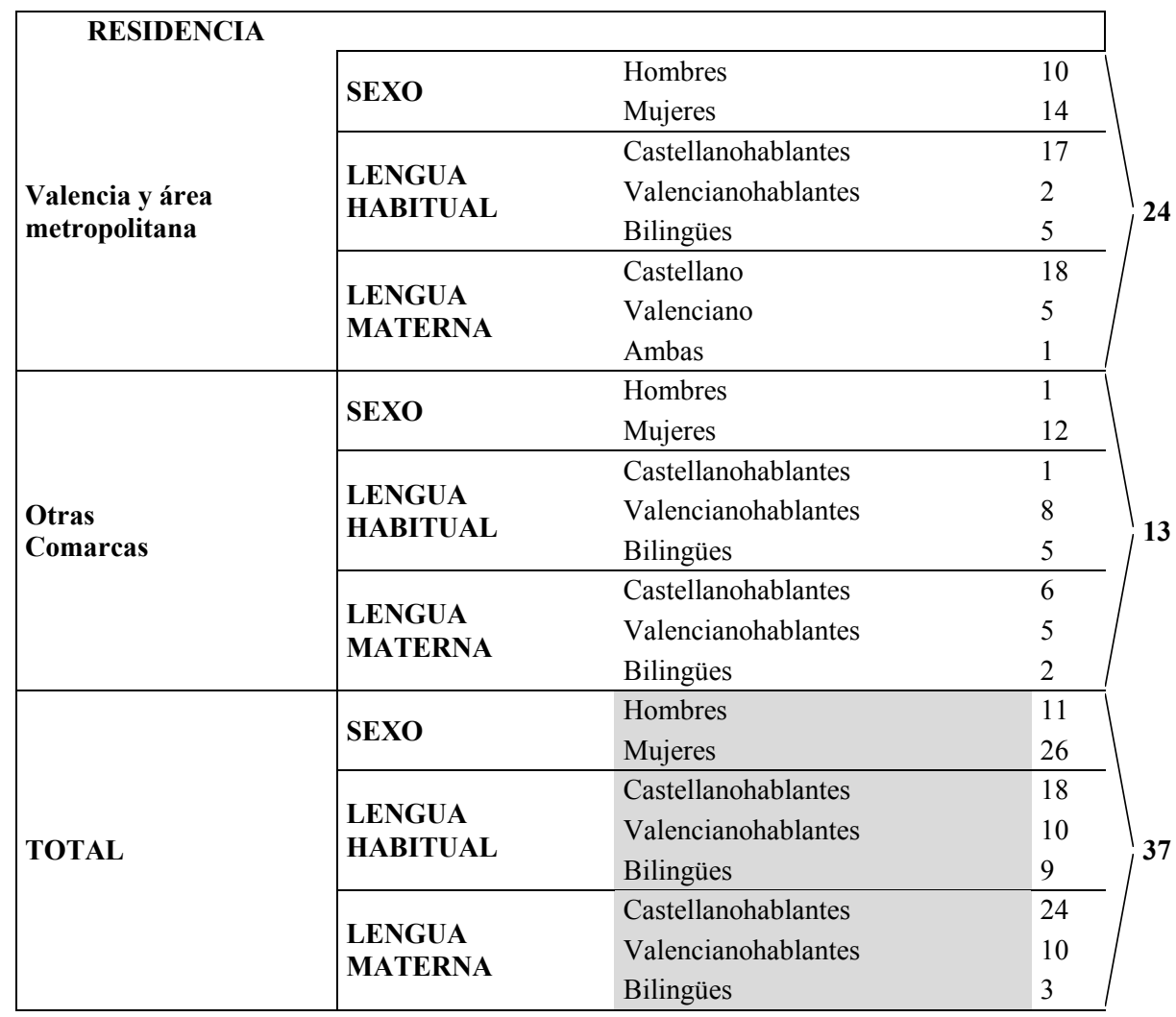




\subsection{La encuesta}

El modelo de encuesta utilizado es idéntico al de la investigación de Buzón et al. (2017: 289). Tras detallarse las variables sociológicas del entrevistado se presentan para valorar un conjunto de 33 pares o binomios ${ }^{4}$ - en escala de Likert de 1 a 7 organizados en dos esferas:

- Dimensión cognoscitiva. Adjetivos relacionados con la competencia (inteligente-no inteligente, seguro de sí mismo-inseguro...) con el estatus (influyente-insignificante, culto-ignorante...), con el prestigio profesional (emprendedor-apocado, identificación jefe y subordinado), con la interacción comunicativa (claro-confuso, convincente-no convincente) y otros que no toman en consideración el diferencial semántico sino ideologías asociadas a las variedades dialectales (valencianista, catalanista, progresista, autonomista) y conciencia sociolingüística - ámbitos de uso de cada modalidad lingüística - (adecuación RTV, escuela, tienda, calle, amigos, casa).

- Dimensión afectiva. Adjetivos relacionados con el atractivo personal (simpático-antipático, divertido-aburrido extrovertido-cerrado...), con el atractivo social (noble-egoísta, generoso-avaricioso...) y grado de identificación con el endogrupo (identificación del locutor con amigos y consigo mismo).

\section{ANÁLISIS ESTADÍSTICOS E INTERPRETACIÓN DE RESULTADOS}

En primer lugar, el conjunto de valoraciones de los jueces sigue una distribución normal que puede describirse como una campana de Gauss; se produce, sin embargo, un valle en la puntuación intermedia y un claro desplazamiento generalizado de las puntuaciones hacia el 5, lo cual se podría interpretar como una tendencia generalizada a evitar la puntuación neutral en beneficio del rasgo positivo de cada binomio.

En términos generales, las diferencias entre las puntuaciones medias muestran significación estadística en el $48,51 \%$ de los casos, valor que indica una significatividad de los resultados relativamente alta (casi en la mitad de los casos se producen diferencias significativas de puntuaciones medias) ${ }^{5}$.

\footnotetext{
${ }^{4}$ El rasgo positivo o negativo de los pares se ha presentado de manera aleatoria a ambos extremos de la escala. Con ello se inhibe la asociación del carácter positivo o negativo a uno de los extremos.

${ }^{5}$ Entre los binomios que no presentan significación estadística en las diferencias de medias (deshonesto/honesto, tacaño/generoso, egoísta/noble, hipócrita/sincero, terco/flexible) todos ellos pertenecen a la dimensión afectiva, lo que indica la homogeneidad en la percepción de sentimientos y emociones de los hablantes independientemente de la modalidad lingüística asociada.
} 


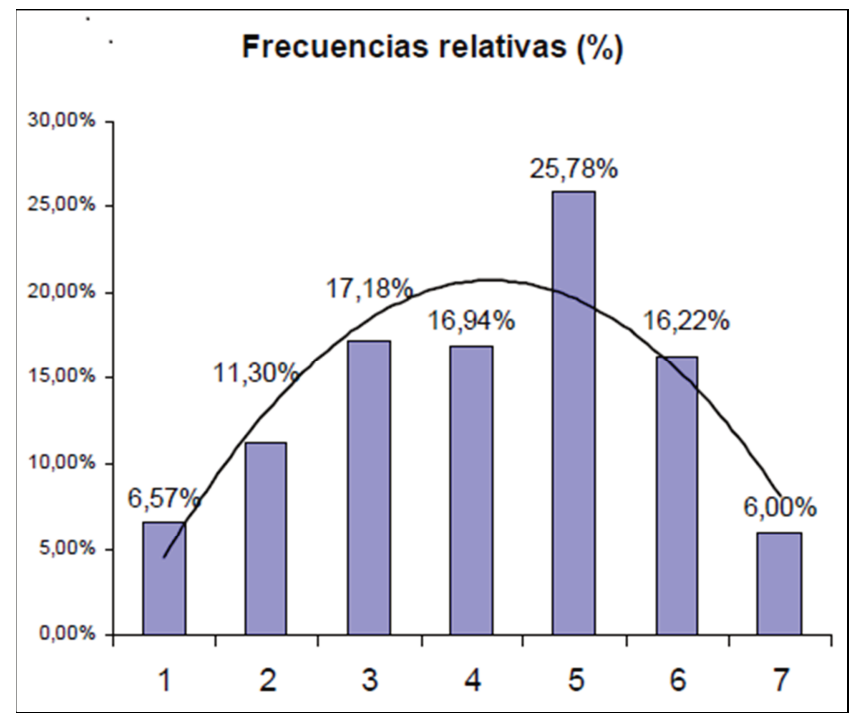

Figura 1. Puntuaciones generales

Ciñéndonos a los objetivos de la investigación y de acuerdo con el primero de ellos, presentamos la categorización social ordenada según puntuaciones medias decrecientes de los estímulos (tabla 2). En las modalidades estándar predominan los atributos de la dimensión cognoscitiva ${ }^{6}$, mientras que en las no estándar son los de la afectiva:

Tabla 2. Organización de los binomios con puntuación media más alta en cada modalidad

\begin{tabular}{|ll|ll|}
\hline \multicolumn{1}{|c|}{ CS } & & \multicolumn{2}{c|}{ VS } \\
\hline Oscuro/Claro & 5,56 & Inseguro/Seguro de sí mismo & 6,03 \\
Maleducado/Educado & 5,33 & Ignorante/Culto & 5,89 \\
Irresponsable/Responsable & 5,33 & No inteligente/Inteligente & 5,67 \\
Hipócrita/Sincero & 5,28 & Maleducado/Educado & 5,67 \\
Vago/Trabajador & 5,17 & Oscuro/Claro & 5,61 \\
No inteligente/Inteligente & 5,11 & Vago/Trabajador & 5,56 \\
Ignorante/Culto & 5,08 & Irresponsable/Responsable & 5,53 \\
Rural/Urbano & 5,06 & Disuasorio/Convincente & 5,31 \\
Deshonesto/Honesto & 5,06 & Callado/Hablador & 5,25 \\
Inseguro/Seguro de sí mismo & 5,03 & Apocado/Emprendedor & 5,14 \\
Callado/Hablador & 4,92 & Deshonesto/Honesto & 5,11 \\
Basto/Refinado & 4,92 & Hipócrita/Sincero & 5,08 \\
Progresista/Conservador & 4,86 & Basto/Refinado & 4,92
\end{tabular}

\footnotetext{
${ }^{6}$ Para facilitar la lectura se han sombreado los binomios de la dimensión cognoscitiva.
} 


\begin{tabular}{|lr|ll|}
\hline \multicolumn{1}{|c|}{ CNS } & & \multicolumn{2}{c|}{ VNS } \\
\hline Orgulloso/Humilde & 5,60 & Avaricioso/Desprendido & 4,97 \\
Callado/Hablador & 5,48 & Presuntuoso/Sencillo & 4,97 \\
Presuntuoso/Sencillo & 5,48 & Ambicioso/Modesto & 4,92 \\
Hipócrita/Sincero & 5,30 & Antivalencianista/Valencianista & 4,78 \\
Antipático/Simpático & 5,15 & Orgulloso/Humilde & 4,67 \\
Avaricioso/Desprendido & 5,13 & Progresista/Conservador & 4,64 \\
Deshonesto/Honesto & 5,13 & Hipócrita/Sincero & 4,44 \\
Ambicioso/Modesto & 4,95 & Callado/Hablador & 4,36 \\
Irresponsable/Responsable & 4,95 & Deshonesto/Honesto & 4,25 \\
Egoísta/Noble & 4,85 & Irresponsable/Responsable & 4,25 \\
Maleducado/Educado & 4,78 & Autonomista/Centralista & 4,11 \\
Triste/Alegre & 4,75 & Gastador/Ahorrador & 4,08 \\
Gastador/Ahorrador & 4,75 & Egoísta/Noble & 4,08 \\
\hline
\end{tabular}

Antes de proseguir y en relación con el análisis de las correlaciones entre las puntuaciones obtenidas por los atributos a nivel global se constatan coeficientes $r$ de Pearson por encima de 0,7 entre buen número de binomios de la dimensión cognoscitiva (no inteligente/inteligente con ignorante/culto, disuasorio/convincente con insignificante/influyente, etc.). De nuevo se hace evidente la mayor homogeneidad social del pensamiento individual (orientado por la creencia colectiva) y la divergencia en el subconsciente afectivo.

Por otro lado, la tabla 3 muestra la categorización social de cada modalidad lingüística por ámbitos. Las variedades estándar predominan en todas las áreas excepto en atractivo personal y social e identificación con amigos. Las modalidades de CS y VS consignan puntuaciones medias prácticamente idénticas en prestigio profesional e identificación personal o con un subordinado, y se destaca VS en competencia personal y estatus. También resulta evidente que la modalidad que mejor favorece el proceso de normalización lingüística y puede ralentizar, neutralizar en el mejor de los casos, el predominio del castellano en la población general (Buzón et al. 2017) es VS.

Tabla 3. Categorización social: jerarquización de las modalidades por puntuación media

\begin{tabular}{|l|ll|ll|ll|ll|}
\hline Competencia personal & VS & $(5,60)$ & CS & $(4,91)$ & CNS & $(3,68)$ & VNS & $(3,26)$ \\
\hline Estatus & VS & $(4,93)$ & CS & $(4,60)$ & VNS & $(2,90)$ & CNS & $(2,89)$ \\
\hline Prestigio profesional & CS & $(4,03)$ & VS & $(4,01)$ & CNS & $(3,00)$ & VNS & $(2,81)$ \\
\hline Interacción comunicativa & VS & $(5,46)$ & CS & $(5,15)$ & CNS & $(3,41)$ & VNS & $(3,24)$ \\
\hline Atractivo personal y social & CNS & $(4,81)$ & VS & $(4,56)$ & CS & $(4,47)$ & VNS & $(4,06)$ \\
\hline Identificación jefe & CS & $(4,33)$ & VS & $(4,28)$ & CNS & $(2,48)$ & VNS & $(2,25)$ \\
\hline Identificación subordinado & VS & $(3,75)$ & CS & $(3,72)$ & CNS & $(3,53)$ & VNS & $(3,36)$ \\
\hline Identificación amigo & VNS & $(4,89)$ & CNS & $(4,70)$ & CS & $(3,58)$ & VS & $(3,33)$ \\
\hline Identificación personal & CS & $(3,36)$ & VS & $(3,33)$ & VNS & $(2,97)$ & CNS & $(2,73)$ \\
\hline
\end{tabular}


Como puede observarse en la tabla 4, el carácter conservador y centralista de los usuarios de CS y CNS discurre en paralelo frente a los valores valencianista y catalanista que se otorga a los hablantes de VS y VNS.

Tabla 4. Tendencias (socio)ideológicas: jerarquización de las modalidades por puntuación media

\begin{tabular}{|l|cc|cc|cc|cc|}
\hline Progresista/Conservador & CS & $(4,86)$ & CNS & $(4,7)$ & VNS & $(4,64)$ & VS & $(4,06)$ \\
\hline Antivalencianista/Valencianista & VS & $(4,89)$ & VNS & $(4,78)$ & CNS & $(3,88)$ & CS & $(3,33)$ \\
\hline Autonomista/Centralista & CS & $(4,75)$ & CNS & $(4,45)$ & VNS & $(4,11)$ & VS & $(3,69)$ \\
\hline Anticatalanista/Catalanista & VS & $(4,44)$ & VNS & $(3,39)$ & CNS & $(3,08)$ & CS & $(2,97)$ \\
\hline
\end{tabular}

La variedad CS se percibe como ligeramente más conservadora y más centralista $\mathrm{y}$, además, como la menos valencianista y catalanista. La modalidad VS, por su parte, es percibida como más valencianista y catalanista, así como la menos conservadora y centralista. Si analizamos el conflicto valenciano/catalán, asunto ampliamente investigado, a pesar de que frecuentemente VS se asocia con catalanismo, la reacción subjetiva hacia esta variedad codificada, normativa y unificadora, ofrece una puntuación muy elevada en las diferentes dimensiones psicosociales, por lo que los intentos de segregar el valenciano central o apitxat (VNS) como lengua propia no cuentan con una fuerza social relevante en la población estudiada. En esta línea cabe señalar que "cuanto mayor es el sentimiento de 'valencianía', más se está a favor de la unidad lingüística, mientras que cuanto más se identifican los sujetos con España, mayor es el sentimiento secesionista del valenciano respecto del catalán” (Agulló 2011: 513).

Retomando ahora el segundo objetivo, los resultados de la tabla 5 muestran una distribución funcional predominante del valenciano en sus dos variedades.

Tabla 5. Ámbitos de uso: jerarquización de las modalidades por puntuación media

\begin{tabular}{|l|ll|ll|ll|ll|}
\hline Adecuación en Radio, TV & VS & $(5,78)$ & CS & $(4,78)$ & CNS & $(2,1)$ & VNS & $(1,86)$ \\
\hline Adecuación en la escuela & VS & $(4,83)$ & CS & $(4,39)$ & VNS & $(2,67)$ & CNS & $(2,63)$ \\
\hline Adecuación para hablar con amigos & VNS & $(5,39)$ & CNS & $(4,83)$ & CS & $(3,08)$ & VS & $(2,81)$ \\
\hline Adecuación para hablar en la calle & VNS & $(5,47)$ & CNS & $(5,1)$ & CS & $(3,11)$ & VS & $(2,83)$ \\
\hline Adecuación para hablar con la familia & VNS & $(5,28)$ & CNS & $(4,83)$ & CS & $(3,36)$ & VS & $(3,19)$ \\
\hline Adecuación para hablar en la tienda & VNS & $(4,78)$ & CNS & $(4,4)$ & CS & $(3,14)$ & VS & $(2,81)$ \\
\hline
\end{tabular}

Las correlaciones entre contextos formales/valenciano estándar (valor instrumental) e informales/valenciano no estándar (valor integrativo, carácter relacional) apuntan a que no se trata de una distribución diglósica (mayor/menor prestigio de una u otra lengua) sino de variación diafásica (adecuación del registro). 
Por último, para atender el tercer objetivo se ha utilizado un modelo estadístico de análisis de la dependencia (ANOVA) ${ }^{7}$. Los resultados, por orden de importancia, se muestran en la tabla 6 .

Tabla 6. Jerarquización de las variables sociológicas por su significatividad estadística

\begin{tabular}{|l|l|l|l|l|}
\hline Global & Lengua habitual & Sexo & Lengua materna & Residencia \\
\hline CS & Lengua habitual & $\begin{array}{l}\text { Sexo } \\
\text { Lengua materna }\end{array}$ & Residencia & \\
\hline VS & Residencia & Lengua habitual & Lengua materna & Sexo \\
\hline CNS & Lengua materna & Lengua habitual Sexo & & \\
\hline VNS & Sexo & Lengua materna & $\begin{array}{l}\text { Lengua habitual } \\
\text { Residencia }\end{array}$ & \\
\hline
\end{tabular}

La variable social 'lengua habitual' aparece asociada al mayor número de diferencias de medias entre las puntuaciones otorgadas a los atributos, especialmente en las variedades de castellano y, en segundo término, de valenciano estándar y castellano no estándar, lo cual representa una valoración de atributos con mayores y más significativas diferencias en función de la lengua habitual del informante. El sexo también influye de manera significativa en las valoraciones de numerosos atributos a nivel global y, particularmente, entre las puntuaciones dadas para la modalidad de valenciano no estándar y, en segundo término, para las dos de castellano. La lengua materna determina un mayor número de diferencias de puntuación significativas para la modalidad de castellano no estándar y la residencia predomina como factor que provoca mayor número de diferencias de puntuación de atributos en la variedad de valenciano estándar.

\section{CONCLUSIONES Y PROSPECTIVA}

El análisis estadístico descriptivo de los datos recabados indica que, tal y como se preveía en las hipótesis iniciales, las variedades estándar - valenciano y castellano - son las más valoradas en la dimensión cognoscitiva: competencia (poder/persona), prestigio profesional, clase social y estatus (poder/grupo), sin diferencias cuantitativas significativas entre ellas. Por su parte, las variedades no estándar - valenciano

\footnotetext{
${ }^{7}$ A través de ANOVA determinamos los factores sociales que se vinculan con un mayor número de diferencias significativas entre medias en las puntuaciones de atributos, en conjunto y por modalidades; es decir, los factores cuyas puntuaciones a determinados binomios han presentado un mayor número de diferencias significativas. Se utiliza el número de atributos que presentan medias significativamente diferentes por modalidad (para un determinado factor social) como indicador de la influencia de ese factor sobre esa modalidad.
} 
y castellano - son las más valoradas en la dimensión afectiva: atracción personal y social, (solidaridad/persona y solidaridad/grupo), grado de identificación (solidaridad/grupo), lealtad y orgullo lingüísticos (solidaridad/grupo), con preferencia hacia el valenciano. Ideológicamente, las variedades de castellano se perciben como más conservadoras y centralistas mientras que las de valenciano son identificadas por los futuros maestros como más valencianistas y catalanistas, percepción más intensa en las variedades estándar que en las no estándar, en cada caso.

En cuanto al segundo objetivo, se constata una variación diafásica en la identificación de los ámbitos formales como más adecuados para la variedad de valenciano estándar y los no formales para la de valenciano no estándar.

Por último, tercero, se observa una alta influencia de la lengua habitual en las diferencias significativas de las puntuaciones otorgadas a un mayor número de atributos, lo cual apuntaría a la importancia de la lengua vehicular de uso habitual como un factor de enorme importancia en la percepción del resto de modalidades disponibles. Según los resultados obtenidos, el resto de variables significativamente influyentes en las diferencias de puntuación a atributos son, en orden decreciente, el sexo, la lengua materna y, en última instancia, la residencia.

En definitiva, los estudiantes de último curso del Grado en Maestro en Educación Primaria (Universitat de València) presentan una percepción del uso (del valenciano) estándar que insiste en el estigma social generalizado de una modalidad de lengua reservada esencialmente para ámbitos formales y vinculada ideológicamente al valencianismo o el catalanismo, si bien es percibida al mismo tiempo como prestigiosa y marca de estatus y poder. El castellano ocupa un lugar preeminente en las percepciones y con cierta capacidad para desplazar al valenciano, por lo que habrá que revisar y reajustar las medidas adoptadas en las últimas décadas. A modo de cierre, es crucial insistir en la necesidad del valenciano como lengua habitual en todas las esferas, dado que ello podría representar un espaldarazo a la mejora de la percepción que de esta modalidad tienen los que, en un futuro no muy lejano, se van a ocupar de la educación de los escolares de primaria. 


\section{BIBLIOGRAFÍA}

Acadèmia Valenciana de la Llengua (AVL) (2005). Llibre Blanc de l'ús del valencià, vol. I: Enquesta sobre la situación social del valencià. Valencia: Acadèmia Valenciana de la Llengua.

Agulló, V. (2011). Análisis de la realidad sociolingüística del valenciano. Papers, 96, 2, 501-514.

Aparici, A. \& Castelló, R. (eds.) (2011). Els usos lingüístics a les universitats públiques valencianes. València: Acadèmia Valenciana de la Llengua.

Buzón, J., Gómez-Devís, M.B. \& Gómez-Molina, J.R. (2017). Actitudes lingüísticas en Valencia y su área metropolitana. Valencia: Tirant lo Blanch.

Casesnoves, R. (2012). El valencià en 25 anys (I). Com són els seus parlants? Revista de Llengua i Dret, 58, 111-136.

Casesnoves, R. \& Mas, J.A. (2016). Usos lingüístics i ideologia: què fan els universitaris valencians? Caplletra. Revista Internacional de Filologia, 60, 113-139.

Casesnoves, R. \& Mas, J.A. (2017). Ideology and language choice. Catalan-speaking university students. Sociolinguistic Studies, 11/1, 107-129.

Giles, Howard \& Ryan, E. (eds.) (1982). Attitudes Towards Language Variation. Social and Applied Context. London: Edward Arnold Publishers.

Gómez-Molina, J.R. (2013). Actitudes lingüísticas en la comunidad de habla bilingüe de Valencia. In B. Montoya \& A. Mas (eds.), Studia Lingüística in Honorem Francisco Gimeno (pp. 375-408). Alacant: Publicacions de la Universitat d'Alacant.

López Morales, H. (1989). Sociolingüística. Madrid: Gredos.

López Morales, H. (1994). Métodos de investigación lingüistica. Salamanca: Ed. Colegio de España.

Mas-Miralles, A. (2018). El valencià segons l'enquesta del SIES de 2015. Treballs de sociolingüistica catalana, 28, 127-145.

Moreno Fernández, F. (1990). Metodología sociolingüística. Madrid: Gredos.

Rojo, G. (1979). Aproximación a las actitudes lingüisticas del profesorado de EGB en Galicia. Santiago de Compostela: Instituto de Ciencias de la Educación, Universidad de Santiago de Compostela. 